\title{
Structure of the Near-Surface Layer of NiTi on the Meso- and Microscale Levels after Ion-Beam Surface Treatment
}

\author{
L. L. Meisner ${ }^{1,2, a)}$, T. M. Poletika ${ }^{1, b)}$, S. N. Meisner ${ }^{1,2, c)}$, \\ A. V. Tverdichlebova ${ }^{1,3, \text { d) }}$, S. L. Girsova ${ }^{1, \text { e) }}$, and I. A. Shulepov ${ }^{3, \text { f) }}$ \\ ${ }^{1}$ Institute of Strength Physics and Materials Science SB RAS, Tomsk, 634055, Russia \\ ${ }^{2}$ National Research Tomsk State University, Tomsk, 634050, Russia \\ ${ }^{3}$ National Research Tomsk Polytechnic University, Tomsk, 634050, Russia

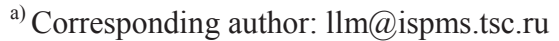 \\ b) poletm@ispms.tsc.ru \\ c) msn@ispms.tsc.ru \\ d) $\mathrm{a} @$ vtverd.ru \\ e) girs@ispms.tsc.ru \\ f) shulepovia@tpu.ru
}

\begin{abstract}
Using the EBSD, SEM and TEM methods, the structure of surface layer of polycrystalline NiTi alloy samples was examined after the modification of material surface by the pulsed action of mean-energy silicon ion beam. It was found that the ion beam treatment would cause grain fragmentation of the near-surface layer to a depth $5 \div 50 \mu \mathrm{m}$; a higher extent of fragmentation was observed in grains whose close-packed planes were oriented approximately in the same direction as the ion beam was. The effect of high-intensity ion beam treatment on the anisotropic behavior of polycrystalline NiTi alloy and the mechanisms involved were also examined.
\end{abstract}

Keywords: NiTi, ion beams, surface layers, martensitic transformations, fragmentation

\section{INTRODUCTION}

To date the ion beam treatment of metallic material surface is a universally adopted method for modification of metal properties. At present, the basic challenge is the creation of a protective barrier in metal surface layers. Due to its unique mechanical properties, titanium nickelide (NiTi alloy) is the most promising metallic material designed for medical applications. It is known that the treatment of NiTi alloy by high-intensity ion beam will cause variation in its phase composition and formation in the near-surface layer of a complex gradient structure [1-3]. High elastic stress levels are found to localize in material zones exposed to the direct action of ion beam as well as in the neighboring zones [4]. The understanding of the exact mechanisms of the observed effects awaits further investigation. The modification of structure depending on the direction of ion beam is poorly understood, which gives no way of deducing the contribution of anisotropy of NiTi to the formation of a complex hierarchic system in the near-surface layer. The goal of the given study is investigation of the regular features of fragmented structure formation in the near-surface layer of NiTi alloy due to the action of silicon ion beam. Of particular interest are also the mechanisms involved, especially in grains variously oriented relative to the direction of ion beam.

\section{EXPERIMENTS}

Ion beam surface modification of the NiTi $\left(\mathrm{Ti}_{49.4} \mathrm{Ni}_{50.6}\right)$ specimens was performed by a DIANA-3 implanter (Tomsk, Russia), using single-ion beam pulses under high vacuum $\left(10^{-4} \mathrm{~Pa}\right)$ conditions in a high-dose ion 
implantation regime. The ion beam processing of the NiTi specimens consisted in Si ion implantation into surface layers. The fluence was $D=6 \cdot 10^{17} \mathrm{~cm}^{-2}$, the average accelerating voltage was $U_{\mathrm{av}}=60 \mathrm{kV}$, and the pulse repetition frequency was $50 \mathrm{~Hz}$. The specimen temperature during the ion implantation was $\leq 373-424 \mathrm{~K}$ [4].

To investigate the microstructure of the surface layer of the methods used EBSD, SEM, TEM by means of machines available at the Center for Collective Use of Scientific Equipment "Nanotekh" of ISPMS SB RAS. For the preparation of thin foils for study by TEM microscope JEM 2100 (JEOL, Japan) used the cross-section samples, obtained by ion thinning with used of EM 09100IS ion slicer (JEOL, Japan). Crystallographic analysis of mutual misorientations fragments was carried out using Wolfram Mathematica (Wolfram Research, USA) with developed for this purpose software module.

\section{RESULTS AND DISCUSSION}

\section{Mesoscopic Inhomogeneous of the Near-Surface Layer}

Using the EBSD technique, it was found that the ion beam treatment of material surface had caused formation of inhomogeneous gradient structure and a change in the phase composition and partial fragmentation of material. In the near-surface layer newly formed areas of martensitic phase B19' and grain fragments 1-15 $\mu \mathrm{m}$ long were found to occur at depths of $1-10 \mu \mathrm{m}$ and 5-50 $\mu \mathrm{m}$, respectively. One further proof of fragmented structure formation was high density of interphase and twin boundaries. The surface layer of the original B2-structure was subjected to modification to a depth of about $60 \mu \mathrm{m}$, i.e. 30 percent of the average grain size. The extent of fragmentation was found to depend on the crystallographic orientation of the original grains in the near-surface layer [4]. Thus the fragmentation was enhanced in grains occurring in the close-packed planes $\{111\}$ and $\{112\}$, which were oriented approximately in the same direction as the incident ion beam.

\section{Crystallographic Analysis of the Fragmentation Mechanism}

Crystallographic analysis was performed for material is as which were identified on misorientation maps from Eulerian angles. Here the purpose was to obtain information about meso-structure formation in the near-surface layer, involving martensitic transition via dominant shear and twinning mechanisms. The analysis suggests that the crystallographic transition B2 $\leftrightarrow$ B19' can be described by operations of rotation of the basis B2; matrices are used to perform rotation and shear for monoclinic distortion of unit cell. To provide for one-to-one correspondence of the B2 and B19'structures in terms of crystallography, we applied the orientation relations obtained by the workers in [5]. Matrices were calculated to perform rotations for each out of twelve possible variants of martensite B19', which are equivalent in terms of crystallography. In addition to the selected orientation relations, we also used experimental data obtained from Euler maps in which crystallite orientations are characterized relative to the selected system of coordinates. Crystallographic parameters were calculated for two groups of matrices, i.e. model and experimental ones; these characterize the orientations of any two crystallites relative to each other. This enabled calculation of indexes of rotation axes and rotation angles around the same axes, which facilitated superimposition of crystallite lattices. The calculated data are listed in Tables 1 and 2. Analysis of calculated data suggests that within the grain having planes oriented in the direction, which is close to that of ion beam, there occur crystallites having structure of B2 type. The rotations of these crystallites, i.e. indexes of rotation axes and rotation angles around the same axes, relative to the original orientation within the grain are, respectively, $\left(119.79^{\circ},[\overline{57.357 .458 .4}]\right) \approx\left(120^{\circ},[\overline{1} \overline{1} \overline{1}]\right)$ and $\left(174.98^{\circ},\left[\begin{array}{lll}3.6 & 0.7 & 99.9\end{array}\right]\right) \approx\left(180^{\circ},[001]\right)$.

TABLE 1. Rotation axes and rotation angles for all the possible intervariant boundaries in martensite B19

\begin{tabular}{cc}
\hline Angles & Axes of Rotation \\
\hline $180^{\circ}$ & {$[001],[011],[0 \overline{1} 1],[\overline{\sqrt{2}} \overline{1} 1],[\sqrt{2} \overline{1} 1],[\overline{\sqrt{2}} 11],[\sqrt{2} 11]$} \\
\hline $90^{\circ}$ & {$[\overline{1} 00],[01 \overline{1}],[100],[0 \overline{1} 1],[011],[0 \overline{1} \overline{1}]$} \\
\hline $120^{\circ}$ & {$[1 \sqrt{2} 0],[\overline{1} \overline{\sqrt{2}} 0],[\overline{1} \sqrt{2} 0],[\overline{1} 0 \sqrt{2}],[10 \overline{\sqrt{2}}],[\overline{1} 0 \overline{\sqrt{2}}],[\overline{1} \sqrt{2} 0],[10 \sqrt{2}]$} \\
\hline
\end{tabular}


TABLE 2. Rotation axes and rotation angles for all the possible combinations of direct and reverse martensitic transformation $\mathrm{B} 2 \rightarrow \mathrm{B} 19^{\prime}$ and $\mathrm{B} 19^{\prime} \rightarrow \mathrm{B} 2$, respectively

\begin{tabular}{cc}
\hline Angles & Axes of Rotation \\
\hline $180^{\circ}$ & {$[001],[100],[010],[110],[101],[011],[\overline{1} 10],[\overline{1} 01],[0 \overline{1} 1]$} \\
\hline $90^{\circ}$ & $\langle 100\rangle$ \\
\hline $120^{\circ}$ & $\langle 111\rangle$ \\
\hline
\end{tabular}

The above values correspond to the values of orientation parameters, which hold good with the proviso that in one of the crystallites a direct martensitic transformation $\mathrm{B} 2 \rightarrow \mathrm{B} 19^{\prime}$ will first occur and then a reverse martensitic transformation $\mathrm{B} 19^{\prime} \rightarrow \mathrm{B} 2$ will take place. As a result, a crystallographic equivalent of the crystallite B2 would form (Table 2). It is also likely that within the analyzed grain there occur are as having the following parameters of rotation: $\left(61.2^{\circ},[\overline{(60.6)} \overline{(54.1)} \overline{(58.2)}]\right) \approx\left(60^{\circ},[\overline{1} \overline{1} \overline{1}]\right),\left(59.9^{\circ},[59.256 .257 .7]\right) \approx\left(60^{\circ},[111]\right)$. This suggests the occurrence of borders of the type $\Sigma=3$, which belong to (112) twins of B2 phase.

Thus, the grains which belong to the family of planes $\{111\}$ and $\{112\}$ and are aligned in the same direction as the incident ion beam, would undergo fragmentation via the mechanism of stress induced direct plus inverse martensitic transformation $\mathrm{B} 2 \leftrightarrow \mathrm{B} 19^{\prime}$.

\section{Meso- and Microstructure}

The above regular features are found to be in conformity with the results obtained in the TEM studies of nearsurface layer defect structure. Thus in the near surface layer at a depth $\leq 5 \mu \mathrm{m}$ the phase B19' internally twinned prevails; this is characterized by the occurrence of nanotwins, which formed due to the deformation.

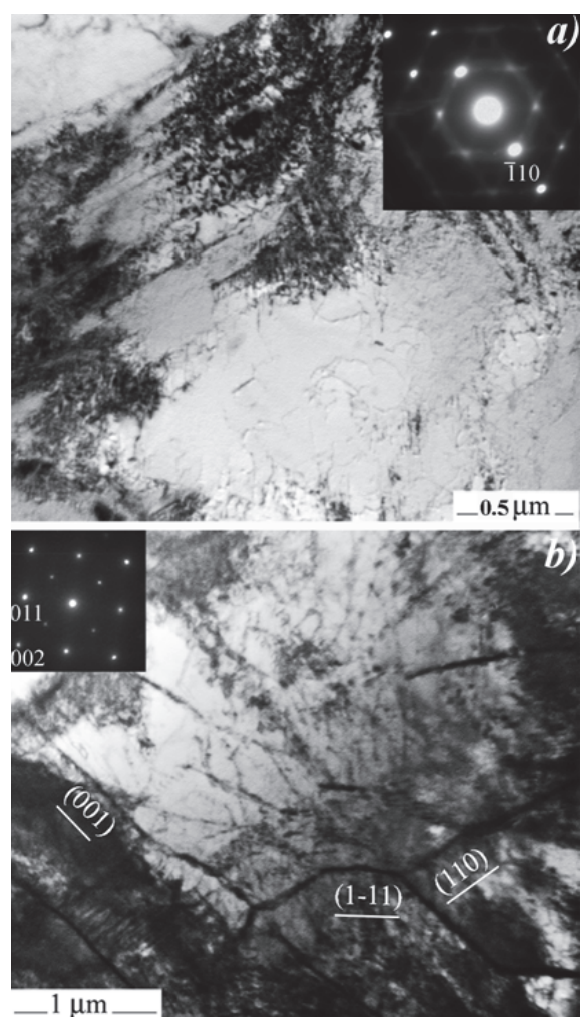

FIGURE 1. The microstructure of NiTi alloy grains having (111) and (100) planes oriented in the direction of the incident ion beam (see (a) and (b), respectively); depth of near-surface layer $\approx 50 \mu \mathrm{m}$ 
The near-surface layer at a depth $\leq 50 \mu \mathrm{m}$ is characterized by significant mesoscopic nonuniformity. The structure of grains is found to depend on their orientation relative to the direction of incident ion beam. Thus crystallite areas of two types are found to occur in the mesostructure of grains having planes oriented in the directions $\{111\}$ and $\{112\}$ and normal to the direction of incident ion beam. These are localization zones composed of B19' phase plates in which self-accomodation takes place as well as areas having B2 phase structure and orientation close to the original one (Fig. 1(a)). The results obtained in the TEM studies are in conformity with the crystallographic analysis data on fragmentation via the mechanisms of direct plus inverse phase transformations $\mathrm{B} 2 \leftrightarrow \mathrm{B} 19^{\prime}$. Characteristically, the fragments have high dislocation density, which hampers structural transformations and thus contributes to the spatial nonuniformity of the mesostructure. On the borders of fragments of all kinds the dislocation density is particularly high. A different situation is observed in grains whose planes, e.g. $\{100\}$, are oriented in the same direction as the incident ion beam. Such grains are found to deform via the mechanism of dislocation glide in the B2 phase to cause formation of localized deformation microbands, which are oriented along the glide planes and have high dislocation density $\left(\sim 5 \times 10^{14} \mathrm{~m}^{-2}\right)$. No traces of $\mathrm{B} 19^{\prime}$ phase were found to occur. Anisotropic fragments are found to form a hierarchic system of low-angle and high-angle boundaries $\left(\leq 3^{\circ}\right.$ and $\approx 60^{\circ}$, respectively). The planes of anisotropic fragments location are of the types $\{001\},\{110\}$ and $\{111\}$, which agrees with the data on the evolution of NiTi structure by severe plastic deformation to cause stabilization of the B2 phase [6]. Due to high gradients of defect density and lattice curvature-torsion, in some fragments dynamic recrystallization will occur (Fig. 1(b)). The development of recrystallization processes is also favored by the enhancement of volume diffusion in elastic strain fields and pulsed irradiation-induced temperature increase. The above results check well with the previously obtained evidence that the anisotropy of NiTi alloy plays a significant role in the development of deformation structure [7].

\section{CONCLUSIONS}

The near-surface layer of NiTi alloy was subjected to ion beam irradiation. Analysis of as-modified structure of the near-surface material layer suggests a high extent of mesoscale structural inhomogeneity, which is due to a wide spectrum of relaxation and accommodation deformation mechanisms acting in the fields of high internal stresses. The prevalent deformation mechanisms are martensitic transformation, formation of localized deformation bands and deformation twinning. Fragmentation also occurs within the grains of near-surface layer; the extent of fragmentation depends on the crystallographic orientation of grains with respect to the irradiated material surface. Crystallographic analysis was made of fragments orientation relative to one another. This enabled tracing the probable routes of fragmentation in the course of direct plus inverse deformation-induced martensitic transformations $\mathrm{B} 2 \leftrightarrow \mathrm{B} 19^{\prime}$, which occurred within variously oriented grains. Using the TEM method, two scenarios of microstructure evolution by fragmentation were revealed: (i) transformation $\mathrm{B} 2 \leftrightarrow \mathrm{B} 19^{\prime}$ within the grains having plane orientation of the type $\{111\}$, i.e. normal to the irradiated surface; (ii) dynamic recrystallization within the grains having plane orientation of the type $\{100\}$, i.e. in the direction of the incident ion beam.

The work was performed within the Program of basic scientific researches of the State Academies of Sciences for 2013-2020.

\section{REFERENCES}

1. L. Meisner, V. Sivokha, A. Lotkov, et al., Physica B 307(1-4), 251 (2001).

2. J. Mayer and M. Nastasi, Ion Implantation and Synthesis of Materials (Springer, 2006).

3. S. Dhara, Rev. Solid State Mater. Sci. 32(1-2), 1 (2007).

4 S. Meisner, Izv. Vuzov. Fizika 57, 108 (2014).

5. O. Matsumoto, S. Miyazaki, K. Otsuka, et al., Acta Metall. 35, 2137 (1987).

6. R. Kocicha, M. Kursab, I. Szurmanb, et al., J. Alloys Compounds 509, 2716 (2011).

7 J. Pfetzing-Micklicha, R. Ghislenib, T. Simona, et al., Mater. Sci. Eng. A 538, 265 (2012). 